\title{
Relaciones interpersonales, elección entre alternativas y resolución de conflictos
}

\author{
Montserrat Payá
}

\section{$\mathcal{D} \mathcal{M a}$}

Con la resolución de conflictos se pretende abarcar el conjunto de técnicas, recursos y estrategias orientadas a la superación de situaciones problemáticas, estableciéndose las fases del procedimiento a seguir en su aplicación en el aula. Asimismo, se presentan ejemplos de actividades dirigidas en especial a los alumnos del primer ciclo de la Educación Secundaria. Además, se ofrecen prototipos de actividades para otros tramos educativos.

\section{INTRODUCCION}

Según E. Guisán ${ }^{1}$, lo moral es todo o está en todas partes siempre que haya seres sintientes. La vida en interrelación, en comunidad, conlleva que entremos en contacto con realidades, opiniones y sentimientos, que pueden estar en desacuerdo con nuestra propia interpretación o que, sencillamente, las vivenciamos como diferentes. En ambos casos, nos situamos ante una fuente de conflicto, real o potencial. Asimismo, cada día nos vemos en la obligación de tomar un número indefinido de decisiones, de desigual relevancia, pero que, de cualquier modo, nos pueden plantear dudas e interrogantes acerca de su idoneidad.

La resolución de conflictos intenta dar respuesta a estas, y otras, exigenciás, proporcionando un método que no es sino un proceso de pensamiento. Su finalidad es ofrecer unas pautas de reflexión y valoración -en las que el sentimiento, el área afectiva, ocupa un lugar destacado-, para analizar y resolver cualquier situación problemática o de toma de decisión. Su fundamento teórico cabe buscarlo tanto en planteamientos directamente vinculados a la Educación para la $\mathrm{Paz}$ y los Derechos Humanos, como en enfoques psicológicos constructivistas. La explicación que aquí se detalla ha intentado encaminar estas aportaciones hacia la práctica educativa en el ámbito de la educación moral y ética. 


\section{¿QUE ES LA RESOLUCION DE CONFLICTOS?}

La resolución de conflictos es una expresión que abara todo el conjunto de técnicas, recursos, estrategias, etc. orientadas a la superación de situaciones problemáticas, entendiendo por tales las que se originan en la relación interpersonal o suponen un problema individual de elección entre alternativas. En muchas ocasiones, el conflicto no radica tanto en la situación concreta como en el sentimiento interno de incerteza que provoca, por el hecho de no saber exactamente cuál es la mejor respuesta. Otras veces, no existe expresamente el conflicto -es decir, reconocido como tal por todas las partes implicadas-, sino tan sólo la percepción de conflicto - una parte considera que lo hay o que puede haberlo. Finalmente, cabe tener presente también que hay problemas sin solución o que, al menos, ésta no depende sólo de nuestros esfuerzos.

Un factor previo y fundamental a la hora de analizar este tipo de experiencias es la manera de situarse ante ellas. Siguiendo a R. Grasa (1991), dos son las formas óptimas de abordar esta tarea: la negociación y la colaboración ${ }^{2}$. La primera consiste en defender los propios intereses o puntos de vista, teniendo también en consideración los de las otras partes implicadas. A ese efecto, se intenta llegar a un punto intermedio aceptado por todas las partes, para el que habrán tenido que ceder de forma cuidadosamente equitativa. La segunda colaboración - supone un paso adelante en lo que se refiere a la educación moral y en valores. Se trata de conseguir los propios deseos al mismo tiempo que se intenta que también lo consigan las otras partes. Para ello, el camino es construir, entre todas las posturas, la alternativa que beneficia a todas ellas. Según Grasa (1991) es también «el enfoque menos usual» ${ }^{3}$.

Partiendo de este requisito actitudinal previo - la colaboración o, en su defecto, la negociación-, se puede abordar ya la resolución de conflictos. Un procedimiento ${ }^{4}$ puede ser el siguiente:

1) Definir el problema y ser consciente de los sentimientos: $A$ veces, resulta especialmente complicado, porque parece ser otro, consta de varias partes, es diferente en función de la persona que lo analiza... Por lo tanto, la primera tarea será especificar exactamente en qué consiste el conflicito, teniendo presente, sin embargo, que esa es nuestra percepción. Para ello, es fundamental reconocer los sentimientos que tal situación provoca en la persona o personas implicadas.

2) Describir las características del problema: El paso siguiente consiste en explicarlo detalladamente, es decir, en enunciar todos aquellos elementos que, de una manera u otra, se relacionan con el problema: cuándo ha pasado o suele pasar y, en ese caso, con qué frecuencia; a propósito de qué motivo o hecho; a quién o a quiénes afecta; qué razones se aducen; qué actitudes se adoptan; qué respuestas se dieron; cómo lo valoran la persona o personas implicadas; qué opina acerca de todo este conjunto cada una de las personas que analizan el conflicto, etc.

3) Elaborar soluciones o alternativas: Sin especificar los detalles y al igual que se hiciera una lluvia de ideas, se pasa a buscar todas las soluciones posibles a la situación analizada. En este momento lo que más importa es la productividad, es decir, encontrar el mayor número posible de alternativas, incluso si resultan fantásticas o parecen imposibles. Se necesitan todas las propuestas, sin entrar aún en valoraciones.

4) Evaluarlas y seleccionar la mejor ${ }^{5}$ : Después, se tiene que valorar cada solución. Para ello, se pueden presentar tres aspectos a tener en consideración: 
Adecuación a la realidad, es decir, si es fácil o no llevarla a cabo; ventajas (a quién beneficia, qué consecuencias positivas puede provocar); inconvenientes (si perjudica a alguien o puede suponer algo negativo). En base a esto, se selecciona la mejor alternativa. Para ello, todo el grupo, sean partes afectadas o no, tiene que estar de acuerdo con la elección o, como mínimo, aceptar que se pruebe.

5) Explicar la realización de la solución: Se trata de profundizar en la propuesta elegida, elaborando un completo plan de acción para llevarla a cabo. Así, se ha de determinar el lugar y momento más adecuados para iniciar la aplicación de la solución y justificarlo con razones; cómo introducir el tema; qué se tiene que decir o hacer exactamente; qué actitud se debe adoptar (confianza, firmeza, etc.); y todos aquellos elementos relevantes para cada situación en concreto (duración, colaboración de otras personas, etc.).

6) Valorar la aplicación de la solución: Una vez aplicada la alternativa durante el tiempo que se consideró conveniente, se han de dedicar unos momentos para reflexionar sobre cómo se aplicó, teniendo como punto de referencia el guión elaborado en el punto anterior. Lógicamente, se comentará también el resultado de la solución, pero sin dejar que eso sea la cuestión fundamental, porque el éxito o el fracaso no depende sólo de la solución ni de la persona o personas que la aplicaron, sino de multitud de factores, a veces, difíciles de reconocer. También es conveniente reservar un espacio de tiempo para pensar cómo afecta ahora el problema - en el caso de que persista - y volver a realizar todo el análisis, si se considera necesario.

\section{UNA PROPUESTA DE ACTIVIDAD}

El ejemplo que ahora presentaremos - diseñado para $8 .^{\circ}$ de E.G.B. y aplicado en grupos de $7 .^{\circ}$ y $8 .^{\circ}$, supone un segundo paso en la aplicación de la estrategia de resolución de conflictos en el aula. Previamente, se habían dedicado dos sesiones de trabajo, estructuradas de la siguiente manera.

En primer lugar, se profundizó en el concepto de «problema», resaltando la vertiente afectiva de este tipo de situaciones y el sentimiento de indecisión e incertidumbre que suscitan al tener que elegir entre varias alternativas e ignorar - al menos, en un primer momento- cuál sería la más adecuada. En la segunda sesión, se presentó y explicó la técnica, con los pasos que aquí se han detallado. Cada una de las fases, además de la explicación, contaba con un texto literario donde se ejemplificaba el momento descrito, sirviendo como modelo. Por último, cada paso concluía con unas cuestiones planteadas al alumno/a para que fueran contestadas a partir de un problema personal que hubiera decidido voluntariamente analizar. El objetivo no era otro que aplicar la técnica, punto por punto, a un conflicto real. La metodología que se utilizó fue individual y colectiva - pequeño y gran grupo- indistintamente. Si bien el apartado dedicado a la aplicación de la técnica fue elaborado inicialmente para realizarse de forma individual, en la práctica se optó también por la aplicación colectiva. La sesión concluía, en el supuesto de realización individual, con la exposición y comentario voluntarios a todo el grupo-clase del trabajo personal.

Pasamos, finalmente, a describir la propuesta de actividad que constituye también un ejemplo de cómo vincular otras áreas -en este caso, la lengua y la literatura-, en el desarrollo de contenidos éticos: 


\section{Tú puedes encontrar una solución}

En las páginas anteriores hemos explicado un poco en qué consiste la resolución de problemas. Ahora te proponemos que lo apliques al problema que plantea este texto:

«Lo que encontraba más penoso de todo era que no siempre fuera capaz de aguantarse las lágrimas cuando los granujas le atacaban fuerte. Por más esfuerzos que hiciera por aguantárselas, al cabo de un momento los ojos le brillaban y después se le iban inundando. Y cuando las lágrimas comenzaban a salir, entonces sí que se lo pasaban bien, los granujas. En ese momento solían reir como locos, golpeándose las rodillas, señalándolo con el dedo y gritando para que todo el mundo se percatase de veras del hecho extraordinario que pasaba.

- ¡Pero miradlo, el mocoso! ¡A Ahora se nos mea por los ojos!

Gritaban cosas así y seguro que continuarían haciéndolo cada día de ahora en adelante. Imaginarlo resultaba una cosa amarga».

(Traducción de: FRICK, L. (1985, 2. ${ }^{\circledR}$ Ed.): L'amenaça. Barcelona: Edicions La Magrana. Col. L'Esparver, n. ${ }^{\circ}$ 18. pp. 26-27).

El chico protagonista se llama Martín. Tiene doce años y, junto a su familia, se acaba de mudar a una nueva ciudad. Ahora le has de intentar ayudar.

a) Explica, según tu opinión, cuál es el problema de Martín. Describe también cómo se debe sentir.

b) Esta situación:

b.1. ¿Afecta sólo a Martin o a más gente? En este último caso, ¿a quién?

b.2. ¿Piensas que le pasa muy a menudo? ¿Por qué sí o por qué no?

b.3. ¿Por qué motivos crees que le pasa?

b.4. Explica que debe pensar Martín a propósito de esto.

b.5. Imagina cómo los compañeros de Martín justificarían su actitud (cuáles serían las opiniones, las razones, etc. que alegarían).

b.6. ¿Y tú qué opinas?

c) Escribe, brevemente, todas las soluciones que puedas imaginar al problema de Martín.

d) Además valóralas, según la facilidad para llevarlas a la práctica y sus ventajas e inconvenientes.

e) Según las valoraciones que acabas de hacer, ¿cuáles serían las mejores soluciones? Explícalas ahora con más detalle, como si las tuvieras que aplicar.

f) ¿Crees que, de verdad, estas propuestas resultarían y que se solucionaría el problema de Martín?

g) ¿Y qué te parece este método para resolver problemas? ¿Piensas que nos puede ayudar? ¿Por qué?

El objetivo final de esta actividad era conseguir la consolidación de la técnica trabajada, la resolución de conflictos. Ello implicaba que fuera interiorizada por los alumnos/as y transferida a todas aquellas situaciones, de carácter personal, vivenciadas como problemáticas. Para ello, se partía de un conflicto, sino real, sí directamente relacionado con los intereses del grupo-clase, como puede serlo un caso de marginación.

La aplicación de la actividad resultó especialmente interesante, sobre todo por lo que hace referencia a la percepción del conflicto. Fue muy sugerente observar cómo la definición del problema que afectaba a Martín, el protagonista de la novela, variaba, no ya en las diferentes aulas donde se aplicó 6 , sino dentro de un mismo grupo. Así, a modo de ejemplo, hubo una clase donde no se verbalizó en ningún momento que el problema fuera la marginación que sufría Martin, sino el no saber hacer frente a las situaciones injustas que le provocaban los otros chicos; su timidez, falta de confianza e, incluso cobardía; el comportamiento del grupo agresor que carecía de toda lógica, justificación y fundamento; etc. Igualmente, se pudo observar cómo la aplicación de la activi- 
dad de forma oral, colectiva y guiada por la maestra, a partir de dos lecturas del texto - una colectiva y otra individual-, redundaba en una sesión mucho más participativa y amena, que no su aplicación en pequeño grupo o individualmente.

Para concluir la actividad, se preguntaba a los alumnos y alumnas su opinión en torno a la técnica en cuestión, a partir de la última pregunta planteada. Se pretendía, por una parte, que la valoraran críticamente y que comentaran su validez para resolver las situaciones conflictivas cotidianas. A este respecto, resaltaban de forma especial su conexión con la realidad, unida a su carácter práctico, características que acostumbraban a poner de relieve en lo que se refiere a la evaluación del área de educación moral y que resultaban determinantes. La actividad ${ }^{7}$ se podía finalizar también iniciando un diálogo acerca del problema de la marginación entre iguales, la dificultad o no de hacer nuevas amistades, la tipología de reacciones ante una persona nueva en el grupo; etc., e incluso, realizar la transferencia hacia el problema de la marginación y discriminación en la sociedad actual.

\section{ORIENTACIONES PRACTICAS}

Un primer interrogante que puede surgir a la hora de plantearse la aplicación de la estrategia de resolución de conflictos en el aula, es su presentación. A este respecto, es aconsejable trabajar primero la definición de problema, a partir de los sentimientos que este tipo de situaciones producen, y constatar cómo las reacciones acostumbran a ser diferentes en función de cada persona, del tipo de problema, de las circunstancias, etc., pudiéndose abrir una vía para la comprensión interpersonal. Asimismo, es aconsejable presentar los problemas de forma positiva, es decir, poniendo de relieve que se pueden solucionar.

Por otra parte, es conveniente plantear la técnica como un posible recurso a utilizar en situaciones de conflicto o de indecisión, que garantiza pensar en profundidad acerca de la mayoría de los factores que están presentes ${ }^{8}$. Igualmente, se puede hacer mención de la importancia de enfocarlos de forma cooperativa, es decir, buscando la mejor solución para todas las personas afectadas y pidiendo la colaboración de los demás, cuando la situación así lo permite. Esta introducción a la estrategia debe ser adaptada en función de la edad de los alumnos y alumnas con quienes se trabaja, y puede requerir dos o más sesiones.

Otro aspecto fundamental es el que se refiere al contenido de los problemas que se presenten para trabajar. Con independencia de la edad, esta estrategia se puede aplicar tanto a un problema real del grupo o de una parte de sus componentes, como a una situación externa al grupo, pero que afecta a uno de sus miembros y lo presenta para que aquél le ayude a solucionarlo. Asimismo, se pueden presentar también problemas hipotéticos, siempre que conecten con las necesidades e intereses de los alumnos/as. En el caso de trabajar con problemas surgidos del grupo-clase, es imprescindible asegurar siempre que haya un clima de respeto. A ello puede ayudar el hecho de separar persona y problema, abordar los conflictos de forma positiva - confiando en su solución-, y hacerlo, además, cooperativamente- con la implicación de todo el grupo-. Las tutorías y las asambleas constituyen un excelente marco.

Finalmente, resta decir que se ha de estimular y motivar la aplicación de la solución, porque es con ella como la técnica cobra pleno sentido. Así, y por lo que se refiere a conflictos reales - personales o de grupo-, se pueden dedi- 
car unos minutos en clase a realizar la evaluación, recordando que se comentará cómo se llevó a cabo, es decir, si se siguió el plan de acción detallado, y sin dejar que la valoración de la solución, ocupe un lugar preeminente.

El papel del educador/a consiste básicamente en orientar y estimular todo este proceso. Ha de enseñar cómo pensar, es decir, cómo analizar un problema, y no qué pensar, cuál es la mejor solución. También ha de prestar especial atención a las cuestiones afectivas que, a veces, pueden surgir. Un clima de respeto y seguridad es básico para abordar tareas de este tipo, pero también es cierto que mediante este tipo de trabajo se pueden facilitar e incrementar tanto la confianza personal como la grupal.

Pasamos, en último lugar, a proponer dos actividades a propósito de la técnica aquí desarrollada, para los cursos de $6 .^{\circ}$ y $2 .^{\circ}$ de E.G.B. respectivamente:

\section{Ayudemos a Nuria}

Con tu grupo tenéis que solucionar el problema de Nuria. Para ello, recordad los pasos que se pueden seguir para resolver problemas. Pensad que vuestra tarea principal es estudiar en equipo la situación y proponer al menos una solución que pueda ayudar a Nuria.

Nuria tiene doce años. Es simpática con sus amigos y amigas, aunque también es muy vergonzosa. El otro día un grupo de niños se metió con ella y no tuvo valor para contestarles. Su amiga, Laura, se ha enterado y se ha enfadado con ella porque no se defendió. Le ha dicho que no volverá a confiar en ella.

- ¿Cuál es el problema de Nuria?

$-¿$ Cuáles son sus causas?

- ¿Qué podría hacer Nuria para solucionar su problema? ¿Cuál es la solución mejor y más realista?

¿Qué puede hacer su amiga Laura y los otros compañeros para ayudarla?

Condiciones de aplicación: Se puede dividir la clase en grupos de tres o cuatro alumnos/as. Una vez contestadas las preguntas, se exponen y anotan todas las soluciones. Posteriormente, se analizan las ventajas e inconvenientes de cada solución, para elegir la más adecuada y detallar cómo se podría llevar a cabo.

Los amigos de Carlota.

Mireia, Genís y Carlota han quedado para jugar en casa de Mireia.

(Dibujo de Mireia): Mireia es una niña muy ordenada y tiene mucho cuidado de sus cosas.

(Dibujo de Genís): Genís es un poco desordenado.

(Dibujo de la situación): Carlota se da cuenta de que Mireia no le quiere dejar sus cosas a Genís.

Genís se enfada y no quiere jugar con Mirella

Carlota no sabe qué hacer para que al final no se enfaden.

Ayudemos a Carlota a resolver el problema. Para hacerlo, contesta las siguientes preguntas:

- ¿Cuál es el problema?

- ¿Por qué crees que pasa esto?

- Busca posibles soluciones.

- Escoge la mejor solución pensando en lo que pasará después.

La mejor solución es.

Si Carlota sigue esta solución, el problema se resolverá porque

Condiciones de aplicación: La actividad se puede enfocar de forma coletiva, buscando así la mejor solución entre todos. Igualmente, se les puede preguntar cómo se deben sentir los tres protagonistas; si les ha pasado alguna situación similar; etc. Una vez elegida la solución, se puede pasar a preparar el guión que ayude a su puesta en práctica. La actividad se puede finalizar con una representación que comprenda desde el enfado hasta la aplicación de la solución. 
Notas

${ }^{1}$ GUISAN, E. (1986). pág. 25.

${ }^{2}$ Otras posibilidades son la competencia, la negación y la acomodación. V. GRASA, R. (1991). pp. 105 y ss.

${ }^{3}$ GRASA, R. (1991). pág. 106.

${ }^{4}$ El método que aquí se detalla constituye tan sólo una de las posibles formas de aplicar la resolución de conflictos. Por consiguiente, puede ser modificado y adaptado en función de las circunstancias.

${ }^{5}$ En edades más avanzadas, por ejemplo, a partir de los doce o trece años, se pueden elegir dos soluciones y someterlas de nuevo al proceso de valoración, para acabar seleccionando una de ellas.

${ }^{6}$ Lógicamente, había diferencias, a nivel de definición del conflicto, entre una clase y otra, pero eran mínimas.

${ }^{7} \mathrm{La}$ duración de la actividad excede una hora de clase. Se puede realizar en dos sesiones, pero con el inconveniente de romper el ritmo y, si ha pasado más de un día, tener que reconstruir de nuevo toda la situación.

${ }^{8}$ En edades más avanzadas, por ejemplo a partir de los trece o catorce años, es muy interesante realizar la valoración crítica de este procedimiento, comentando si ha sido aplicado en situaciones reales y, en ese supuesto, qué dificultades o ventajas han encontrado, cuáles son sus opiniones, etc.

\section{Referencias}

CarriLlo, I. (1991): «Habilidades sociales». En: M. Martínez-Josep M. ${ }^{a}$ Puig (Coord.): La educación moral. Perspectivas de futuro y técnicas de trabajo. Barcelona: Graó I.C.E. Universitat de Barcelona. Col. Materiales para la Innovación Educativa (M.I.E.), n. ${ }^{\circ}$ 4. pp. 138-141.

Cascon, P. Grasa, R. (1988): «Decidir sin marginar». Cuadernos de Pedagogía, 165 (Diciembre, 1988). pp. 17.19.

Fabregas, J. M. GarCía, E. (1988): Técnicas de autocontrol. Madrid: Biblioteca de Recursos Didácticos Alhambra. pp. 21-47i 111-117.

GrasA, R. (1987): «Vivir el conflicto». Cuademos de Pedagogia, n. 150 (Julio-Agosto, 1987). pp. 58-62 . (1988): «Paisaje para una polémica». Cuademos de Pedagogía, n. ${ }^{\circ} 165$ (Diciembre, 1988). pp. 8-12.

(1991): «Resolución de conflictos. Observa cómo discuten o se pelean para decidir cómo negociar». En: M. Martínez-Josep M. ${ }^{a}$ Puig (Coord.): op. cit. pp. $105-112$.

GuIsan, E. (1986): Razón y pasión en Ética. Los dilemas de la ética contemporánea. Barcelona: Anthropos.

Judson, S. (1986): Aprendiendo a resolver conflictos. Barcelona: Lema. (1987): “Cómo hacer para...» Cuademos de Pedagogia, n. 150 (Julio-Agosto, 1987). pp. 36-37.

Michelson, L.; Sugai, D. P.; Wood, R.P.; Kazdin, A. L. (1987): Las babilidades sociales en la infancia. Barcelona: Martinez Roca. pp. 165-170. 


\section{Relaciones interpersonales, elección entre alternativas y resolución de conflictos Montserrat Payá Sánchez CL\&E, 1992, 15, pp. $85-91$}

Resumen: La estrategia de resolución de conflictos pretende sistematizar el proceso de reflexión presente en el momento de analizar una situación que es percibida como problemática y a la que, consecuentemente, se debe plantear una alternativa. Si bien es aplicable tanto a nivel individual como colectivo, es de destacar el carácter cooperativo de la técnica en sí, que apuesta por la construcción de la solución que beneficie a todas las partes implicadas.

Datos sobre el autor: Montserrat Payá Sánchez es licenciada en Pedagogía y miembro del Grupo de Investigación en Educación Moral (G.R.E.M.) de la Universidad de Barcelona. Desde 1989 imparte clases de Etica - niveles $7 .^{\circ}$ y $8 .^{\circ}$ de E.G.B.- en diversos colegios públicos de Cornellá del Llobregat (Barcelona).

Dirección: Departamento de Teoría e Historia de la Educación, Facultad de Pedagogía, Universidad de Barcelona, Baldiri Reixac, s/n 08028 Barcelona. Tel (93) 3333466.

(C) De todos los artículos deberá solicitarse por escrito autorización de CL\&E y de los autores para el uso en forma facsímil, fotocopia o cualquier otro medio de reproducción impresa. CL\&E se reserva el derecho de interponer las acciones legales necesarias en aquellos casos en que se contravenga la ley de derechos de autor. 